\title{
Aplicación de modelos de datos funcionales para el análisis descriptivo de la actividad eléctrica cerebral en estudiantes universitarios
}

Applied Functional Data Models for Descriptive Analysis of Brain Electrical Activity in Undergraduate Students

Recepción del artículo: 15-09-2021 | Aceptación del artículo: 26-01-2022

\section{Camargo-Peña Andres ${ }^{1}$ \\ pena.camargo@unimelb.edu.au \\ https://orcid.org/0000-0002-3644-4864 \\ Sastre-Gómez Luz Viviana ${ }^{2}$ \\ v.sastregomez@unimelb.edu.au \\ https://orcid.org/0000-0002-1748-6436 \\ Urrea, Carlos ${ }^{3}$ \\ urreasamb@gmail.com \\ https://orcid.org/0000-0001-6231-8039 \\ Mongui, Jose ${ }^{4}$ \\ josea.monguif@gmail.com \\ https://orcid.org/0000-0003-2633-5557 \\ 1,2 The university of Melbourne \\ 3, 4 Universidad Los Libertadores}

Para referenciar este artículo:

Camargo-Peña, A., Sastre-Gómez, L. V., Urrea, C. y Mongui, J. (2022). Aplicación de un modelo de datos funcionales para el análisis descriptivo de la actividad eléctrica cerebral en jóvenes universitarios. Revista ConCiencia EPG,

$7(1), 84-100$

https://doi.org/10.32654/CONCIENCIAEPG.7-

Autora corresponsal: Sastre-Gómez, Luz Viviana v.sastregomez@unimelb.edu.au. Melbourne, Australia

\section{Resumen}

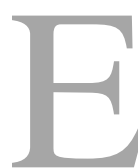

1 presente estudio tuvo como objetivo identificar características de la actividad eléctrica cerebral de estudiantes universitarios expuestos a una tarea de sinceridad y simulación de engaño, utilizando un modelo funcional para el análisis de datos electroencefalográficos. Se realizaron análisis Bspline con el fin de identificar artefactos en las señales eléctricas y posteriormente se realizó un análisis descriptivo de datos funcionales para confirmar indicadores de dispersión y atipicidad de los datos. Los resultados permitieron identificar que durante la condición de engaño la actividad eléctrica cerebral no solo presenta una mayor amplitud en las ondas, especialmente en áreas las áreas frontotemporales, sino que el nivel de dispersión de los datos son considerablemente menores en comparación a la actividad cerebral registrada bajo la condición sin engaño.

Palabras Clave: Análisis de datos funcionales, electroencefalografía, engaño simulado, patrones de actividad cerebral, suavizado B-spline, Emotiv Epoc. 
Abstract

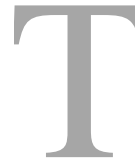

his research aimed to identify characteristics of the brain electrical activity of undergraduate students exposed to honesty and deception tasks, using a functional model for the analysis of electroencephalographic data. B-spline analyses were conducted to determine potential artefacts from electrical signals. Subsequently, a descriptive analysis of functional data was deployed to confirm levels of dispersion and outliers. The results allowed to identify that, during the deception condition, the brain activity not only displays greater amplitude, especially in the frontotemporal areas, but also that the level of dispersion is considerably lower compared to the misleading condition.

Key Words: functional data analysis, electroencephalogram, simulated deceit, brain electrical activity, Bspline, Emotiv Epoc

\section{Introducción}

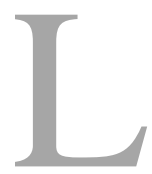

a actividad eléctrica cerebral, ha sido un fenómeno ampliamente estudiado desde las diferentes disciplinas que basan sus conocimientos en los fundamentos empíricos propuestos por la revolución tecnológica en el campo de la neuroimagen (Cortes, García-Hernández, Iribe-Burgos, Hernández-González, Sotelo-Tapia, y Guevara, 2021; Maestú, Quesney-Molina, Ortiz-Alonso, Campo, Fernández-Lucas, y
Amo, 2003;) la cual, es usada para establecer relaciones entre múltiples estímulos que son procesados en las zonas de la corteza cerebral. Así mismo, las mediciones a través de electroencefalograma (EEG) han permitido dar respuesta a preguntas relacionadas con las regiones cerebrales que se activan frente al procesamiento de estímulos externos $\mathrm{y} / \mathrm{o}$ procesos cognitivos relacionados con la interpretación de la información y el momento en el que dichas áreas manifiestan grados de activación cerebral.

Existen diversos dispositivos de electroencefalografía con los cuales ha sido posible evaluar la actividad eléctrica cerebral, entre ellos se encuentra EmotivEpoc, el cual ha demostrado múltiples utilidades en el estudio de las señales eléctricas cerebrales, por ejemplo en un estudio publicado por Ierache, Pereira, Iribarren, y Nervo, (2014), en donde utilizando el equipo Emotiv-Epoc brain computer interface scientific contextual EEG, se encontró que los participantes que fueron expuestos hacia alguna fuente de estimulación, presentaron un incremento considerable en la actividad eléctrica cerebral, ocasionada por la presencia de reacciones emocionales elicitadas por la exposición a un objeto.

El estudio con EEG posee múltiples técnicas aplicables al estudio de los procesos neuronales y los factores fisiológicos subyacentes, una de estas es la neuroimagen funcional, encargada de estudiar las variaciones neurofisiológicas desencadenadas en procesos cognitivos específicos, sin acudir a pacientes con 
lesiones cerebrales, lo cual permite acceder al funcionamiento del cerebro en estado normal, incluyendo los procesos sensoperceptuales, planeación y toma de decisiones, (Rodríguez-Valdez, ManriqueSuárez, Álvarez-Amador, Galán-García, Fernández-García, López-Cánovas, Riverón, Bobes-León, 2008).

Un reciente estudio (Kouhbanani y Arabi, 2021), determinó que las señales eléctricas originadas a través de la comunicación neuronal entre las diferentes áreas especializadas de la corteza cerebral, están asociadas con múltiples funciones cognitivas de orden superior, las cuales representan un acercamiento a la explicación del comportamiento humano, razón por la cual, durante los últimos años, se ha incrementado de manera exponencial el estudio de comportamientos particulares a través de la interpretación de la actividad eléctrica (Kouhbanani y Arabi, 2021). Para ilustrar esta idea, Ortega (2010) afirma que procesos esenciales como la tendencia a mentir, han sido analizados como un fenómeno social que tiene implicaciones en el contexto de las relaciones humanas, sin embargo, a partir de enfoques experimentales ha sido posible identificar la representación neurocognitiva que subyace a este fenómeno social $\mathrm{y}$ psicológico (Bessadok, Mahjoub, y Rekik, 2021).

Según Ekman citado por Monaro, Maldera, Scarpazza, Sartori y Navarin, (2022), el estudio de las emociones y expresión corporal permite un acercamiento al estudio de la mentira, partiendo del punto de que las expresiones faciales de las emociones no están determinadas por parámetros culturales, sino que hay un principio de corte biológico que moviliza el comportamiento. Bajo este principio, la mentira ha sido estudiada a partir de mecanismos conductuales (observación de manifestaciones motoras asociadas a elementos emocionales), o por mecanismos neurofisiológicos (métodos que estudian procesos cognitivos a partir de la actividad eléctrica del cerebro). Este último mecanismo ha sido ampliamente estudiado no solo en el campo de la mentira, sino en todo tipo de actividades de corte cognitivo, las cuales han sido validadas estadísticamente a partir de la implementación de nuevas técnicas de análisis temporal, transformaciones de series de Fourier y Análisis Funcional de datos, los cuales son considerados como uno de los métodos más precisos debido a las técnicas de suavizamiento de los datos (Piñerez, Ramírez, y Escobar, 2017).

Por otra parte, el estudio de patrones asociados a la mentira desde una perspectiva neurofisiológica ha sido fuente de referencia para identificar el grado de validez de los procesos cognitivos implicados en la elaboración de mentiras. Los estudios en EEG, pueden aportar a este conocimiento, ya que registran la actividad eléctrica del cerebro asociada a la toma de decisiones $\mathrm{y}$ otros procesos cognitivos complejos (Berberyan, Van Maanen, Van Rijn, y Borst,2021). Son diversos los equipos que realizan esta actividad, entre ellos se cuenta con el Emotiv-Epoc que entre sus diversos usos puede estar el hecho de considerar la posibilidad de evaluar la verosimilitud del testimonio de un individuo en términos electroencefalógrafos. Generalmente, los 
procedimientos utilizados para medir el grado de veracidad en un evento específico corresponden a la técnica de poligrafía y entrevista cognitiva (Rodríguez-Valdez et al, 2008) pero no hacen énfasis en las respuestas electroencefalográficas, por eso desde equipos como el Emotiv-Epoc es posible evaluar como técnica alternativa complementaria, y quizá como técnica de base para otros procedimientos, el registro de actividad neuronal recolectado para confirmar si los datos obtenidos representan indicadores de procesos cognitivos implicados en elaboración de mentiras.

\section{Evaluación del engaño}

En términos concretos, la mentira puede ser definida como un intento deliberado de ocultar o manipular información factual por medio de verbalizaciones con el fin de crear o mantener una creencia en otra persona (Martínez, 2020). Autores como Ford (1988) y Hala (2001) citados por Castro, López y Morales (2013) concuerdan en que este proceso se da desde la infancia, argumentando que entre los 2 y los 5 años ya existen estrategias de engaño y comprensión de la verdad de modo tal que es posible manipular la información y crear falsas creencias en otros. Adicionalmente, los procesos cognitivos relacionados con la capacidad para detectar y elaborar mentiras son proporcionales al nivel de procesamiento de la información y maduración de estructuras neuronales propios del desarrollo cognitivo del individuo, lo cual indica que el desempeño de estas tareas es mediado por el desarrollo de procesos cognitivos complejos y el lenguaje (De paulo y Kashy, 1996, citado por Castro, López y Morales, 2013).

Los procesos subyacentes a la mentira no solamente han sido estudiados a partir de las respuestas fisiológicas generadas a través de la activación del sistema nervioso periférico, incluyendo respuestas autónomas asociadas con la ansiedad, la expresión facial, gestos y el tono de voz, sino que también mediante el análisis de la actividad eléctrica cerebral derivada de los procesos cognitivos asociados a la elaboración de mentiras (Castro, et al 2013). De acuerdo con Sahakian y Gottwald, (2016), estudios con resonancia magnética funcional han encontrado que individuos que tienden a mentir frecuentemente, presentan una mayor activación neuronal del lóbulo frontal, esto indica que procesos cognitivos asociados a esta área en particular podrían ser evaluados para detectar la presencia de mentiras, (Battista, Otgaar, Mangiulli, and Curci, 2021).

De acuerdo con Gutiérrez, Giner, Quintero, Moret y Moliner (2014), se puede trabajar la detección de mentiras mediante el registro electroencefalográfico con al menos dos electrodos de la onda P300, esta onda está asociada al almacenamiento de recuerdos que mide básicamente el reconocimiento que hace el sujeto a un hecho frente a un estímulo, por lo que éste emite de forma involuntaria una determinada amplitud de la onda, la ventaja de este registro es que los potenciales evocados cognitivos no pueden ser manipulados por el participante, puesto que estas señales no dependen de factores emocionales. 
De acuerdo con lo mencionado, el presente estudio tiene como objetivo identificar características de la actividad eléctrica cerebral de estudiantes universitarios expuestos a tarea de engaño simulado, utilizando un modelo aplicado para el análisis de datos funcionales recolectados a través del equipo de medición electroencefalográfica EmotivEpoc.

\section{Método}

Diseño: La presente investigación tiene un enfoque descriptivo a nivel cuantitativo. Adicionalmente, registros en la actividad eléctrica cerebral fueron recolectados en cada uno de los participantes, tanto en la fase experimental como en la condición control, lo cual según Shaughnessy, Zechmeister y Shaughnessy (2007), permite identificar con mayor precisión el efecto de la variable independiente sobre la dependiente, debido a que los resultados presentan una menor probabilidad en el error de variación en la ejecución de cada participante.

Participantes: Por medio de un muestreo no probabilístico por conveniencia, fueron seleccionados 11 participantes, 7 hombres y 4 mujeres, con edades comprendidas entre los 17 y 22 de edad. Dentro de los criterios de exclusión fueron considerados aspectos relacionados con el consumo de sustancias psicoactivas; alteraciones relacionadas con estados de sueño y/o dolores crónicos; trastornos de ansiedad y consumo de medicamentos que pudiesen afectar el funcionamiento normal de la actividad cerebral.
Instrumentos: Para la recolección de los datos se utilizó el equipo Emotiv-Epoc, brain computer interface scientific contextual EEG, diseñado para recolectar y almacenar el registro bioeléctrico cerebral. Basado en la forma e intensidad de las señales recibidas en cada uno de los 16 canales, el Emotiv-Epoc permite recolectar información sobre la actividad cerebral a partir del procesamiento de la información eléctrica.

Adicionalmente, se aplicó una encuesta demográfica con preguntas sobre datos personales generales como edad, género, ciudad de residencia y lugar de nacimiento, así como preguntas relacionadas con información académica. Subsecuentemente, los participantes recibieron una ficha fotográfica con información detallada sobre un dispositivo electrónico de uso cotidiano (teléfono móvil, computador portátil o tableta), la cual fue utilizada como estímulo para determinar cada una de las condiciones experimentales a las que fueron expuestos los participantes al momento de proporcionar declaraciones verdaderas y falsas de la información suministrada.

También, se llevó a cabo una entrevista compuesta por un conjunto de preguntas estructuradas, relacionadas con las características técnicas de los dispositivos electrónicos seleccionados previamente, las cuales permitieron comparar la información recolectada en cada una de las condiciones. Durante la entrevista fueron evaluadas las preguntas basadas en las encuestas demográficas; la consistencia de las respuestas registradas en cada una de las condiciones 
experimentales y cambios notorios en el comportamiento de los participantes durante la fase de evaluación de engaño.

Cabe resaltar que los instrumentos utilizados durante el estudio cumplieron con los lineamientos generales para la investigación en salud, teniendo en cuenta que los métodos para la obtención de los datos no son invasivos. Por lo cual no se identificaron situaciones de riesgo para la salud física o psicológica de los participantes.

Procedimiento: después de explicar el procedimiento y obtener consentimiento informado por parte de todos los participantes, se realizó una revisión y calibración de los instrumentos utilizados para el registro de la actividad cerebral con el fin de evitar sesgos ocasionados por una adecuación imprecisa de los equipos. Ulteriormente, los participantes fueron dirigidos individualmente a la cámara de Gesell, con el fin de reducir niveles bajos de atención ocasionados por la presencia de distractores en el momento de las mediciones. En la siguiente fase del estudio se instaló el equipo Emotiv-Epoc, identificando la calidad en la conexión de cada uno de los electrodos que conforman el sistema 10-20, específicamente, los electrodos AF3 y AF4 correspondientes a la zona frontal anterior del cerebro; F7, F3, F4, F8, FC5 y FC6 ubicados en la zona frontal del en la zona frontal medial del cerebro.

Inicialmente, los sujetos completaron un formato de encuesta demográfica, incluyendo información verídica sobre sus datos personales y académicos. Por otra parte, cada uno de los participantes seleccionó una ficha fotográfica con información detallada sobre un dispositivo electrónico de su preferencia. Seguido de esto, se les pidió a los sujetos que indicaran las características reales del dispositivo electrónico seleccionado, acorde a la información registrada en la ficha fotográfica. Posteriormente, y con el propósito de medir la actividad cerebral en condición de engaño, se solicitó a los participantes que proporcionaran información completamente diferente a las características reales del mismo objeto seleccionado en la fase inicial. Finalmente, se les solicitó a los participantes completar con información inverosímil, una segunda encuesta con el mismo número y tipo de preguntas de la encuesta inicial. Durante la fase final se realizó un proceso de retroalimentación a cada uno de los participantes, con el objetivo de identificar posibles aspectos emocionales desencadenados durante el procedimiento de evaluación

\section{Resultados}

Pese a que el equipo de medición electroencefalográfica Emotiv-Epoc cuenta con 16 electrodos encargados de recolectar información bioeléctrica, el presente estudio solo analizó información proveniente de 8 electrodos ubicados en las cortezas frontal y prefrontal, ya que según Berberyan et al. (2021), en dichas áreas se llevan a cabo procesos cognitivos íntimamente relacionados con la elaboración de mentiras y engaño. Durante la fase de preprocesamiento, los datos fueron extraídos y transformados a archivos con formato CSV a través del software EMOTIV PRO con licencia 
educativa diseñada para contextos académicos y de investigación. Así mismo, el análisis estadístico de datos funcionales se aplicó un algoritmo de suavización de los datos a través de bases B-Spline, Línea Polinómica, Suave Básica, comúnmente utilizado para la limpieza e identificación de artefactos en las señales eléctricas. Posteriormente, se realizó un análisis descriptivo funcional con el fin de determinar los valores promedio de la actividad eléctrica cerebral, la desviación estándar funcional e identificación de datos atípicos. Según Ramsay \& Silverman (2002), el análisis descriptivo para datos funcionales se debe realizar a partir de la estimación de las siguientes expresiones:

La fórmula para el cálculo de la media funcional es igual a:
$\bar{x}(t)=N-1 \sum_{i=1}^{N} x i(t)$

La fórmula para el cálculo de la varianza funcional es igual a:

$\operatorname{Var} x(t)=(N-1)-1 \sum_{i=1}^{N}[x i(t)-\bar{x}(t)]^{2}$,

La fórmula para el cálculo de la desviación estándar funcional es igual a:

D.E. $(x(t))=\sqrt{\operatorname{Var}(x(t)) .}$

Los análisis fueron realizados utilizando la versión 2.25 del software estadístico R.

\section{Figura 1}

Actividad eléctrica de la corteza frontal anterior, electrodo AF3

Condición A (sin engaño)

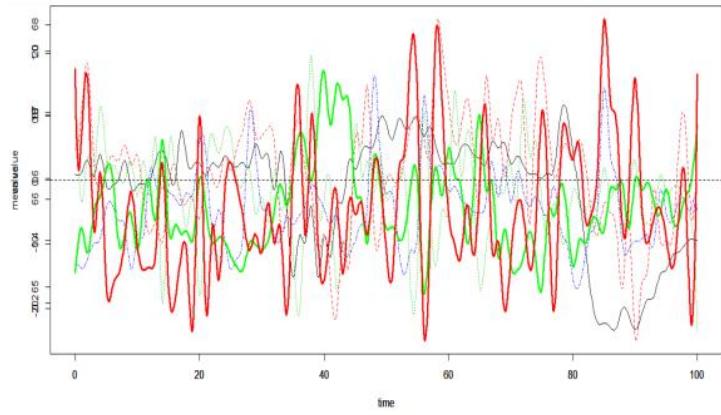

Los resultados obtenidos en el análisis muestran que en la condición $\mathrm{A}$ (sin engaño), el electrodo AF3 presenta un rango de amplitud entre $65 \mu \mathrm{V}$ y $68 \mu \mathrm{V}$, lo cual indica que a pesar de que la polaridad en el conjunto de ondas es positiva, el comportamiento de la señal eléctrica es inestable, presentado ascensos y descensos
Condición B (con engaño)

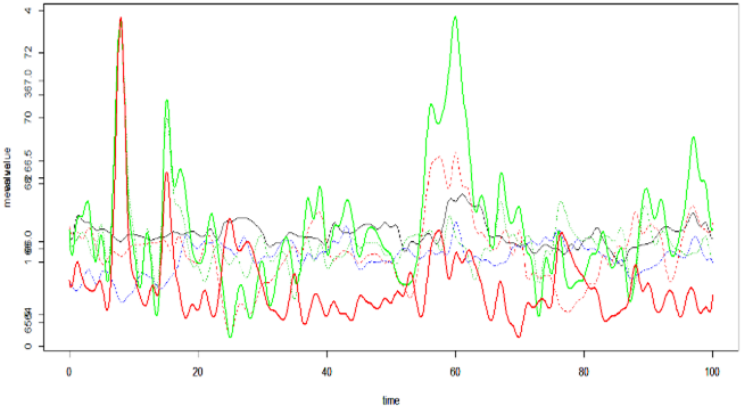

en diferentes puntos. Al analizar la media (demarcada con el color verde) y la desviación estándar (demarcada con el color verde), es posible observar bajos índices de dispersión puesto que los valores asociados a la desviación estándar funcional del electrodo F3 no se aleja demasiado de la media. 
En el análisis de la condición B se observa que el nivel de voltaje aumentó (64 - $72 \mu \mathrm{V}$ ) respecto a la condición A, lo cual indica que el conjunto de ondas mostró mayor actividad eléctrica. Por otra parte, el comportamiento de la actividad eléctrica no presenta índices elevados de ruido en comparación a la situación A, indicando que la onda es estable y continua a lo largo del eje x. De igual manera, se puede identificar que la media y desviación estándar presentan mayores índices de dispersión en comparación a la condición A, mostrando un patrón de dispersión estable a lo largo de la medición del electrodo.

\section{Figura 2}

\section{Actividad eléctrica de la corteza frontal anterior, electrodo AF4} Condición A (sin engaño)

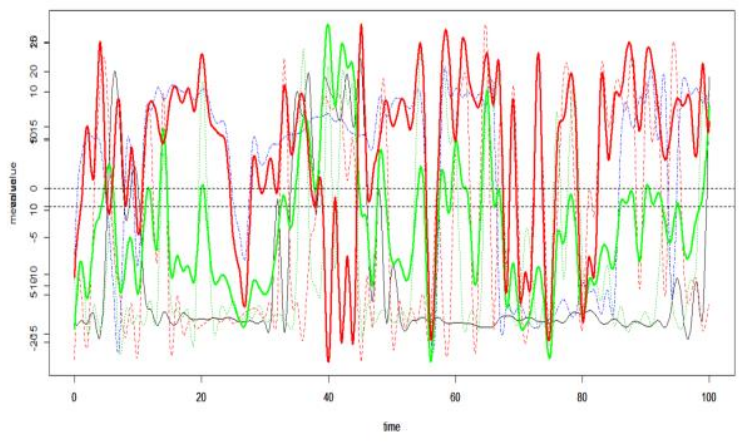

En la Figura 2, se representa la señal eléctrica tomada por electrodo AF4. En la condición A es posible observar que la amplitud de la onda se encuentra en un rango -20 a $20 \mu \mathrm{V}$, compartiendo polaridad positiva y negativa. Por otra parte, los datos presentan alto nivel de rugosidad a través de las observaciones, la desviación estándar (representada con la línea roja), se encuentra en algunos casos al mismo nivel de la media y en otros de hecho muy por encima, lo cual muestra que el nivel de variación no depende de posibles artefactos aleatorios, sino que los datos se siguen teniendo altos niveles de dispersión en esta área específica.

Respecto a la condición B permanece entre $-20 \mu \mathrm{V}$ y $20 \mu \mathrm{V}$, en este caso, no se
Condición B (con engaño)

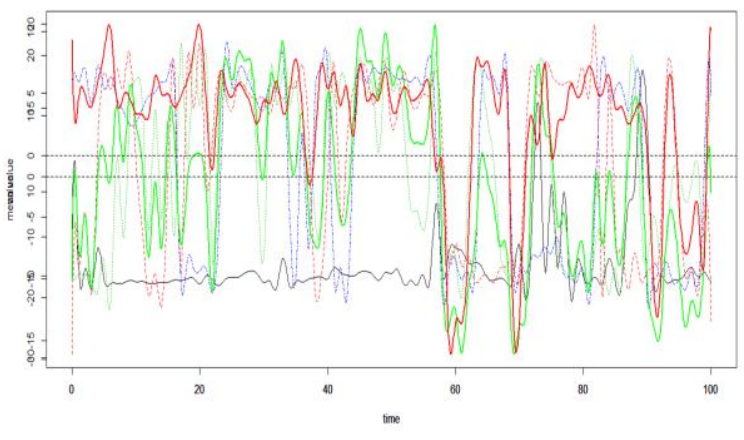

observan variaciones drásticas en el comportamiento de la señal eléctrica, ocasionado por el cambio de condición de engaño, sin embargo, cabe resaltar que la mediana ya no es tan ambivalente, por lo cual se ve una mayor tendencia hacia la polaridad positiva. En cuanto a la rugosidad de la curva, se mantienen niveles altos con comportamientos fluctuantes de la desviación estándar, indicando que hubo una mayor variación de la actividad eléctrica de la corteza frontal anterior en la condición de engaño. 


\section{Figura 3}

Actividad eléctrica de la corteza frontal, electrodo F7 Condición A (sin engaño)

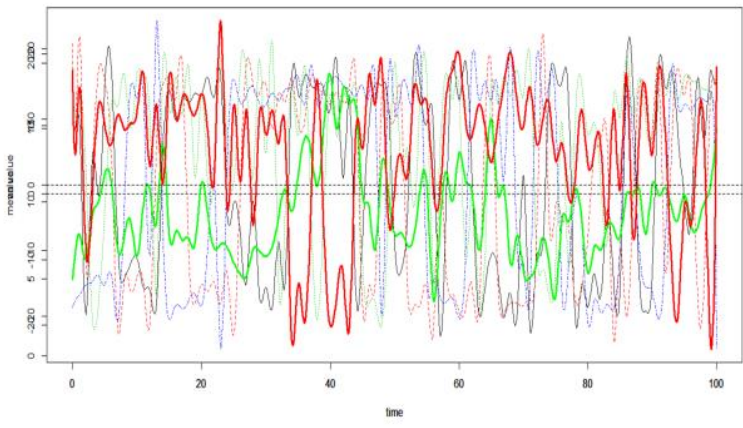

La Figura 3 muestra que para la condición A, la amplitud de las ondas varía de polos positivos a negativos con rangos de amplitud de - 20 a $20 \mu \mathrm{V}$. El comportamiento de la señal a través de la longitud muestra altos índices de dispersión, sin embargo, la rugosidad de los datos tiende a estabilizarse permitiendo identificar el comportamiento estable de las ondas. La media funcional se encuentra entre valores que van de $-10 \mu \mathrm{V}$ a $10 \mu \mathrm{V}$, mientras que la desviación estándar presenta valores entre $0,5 \mu \mathrm{V}$ y $15 \mu \mathrm{V}$ confirmando índices elevados de dispersión de los datos.

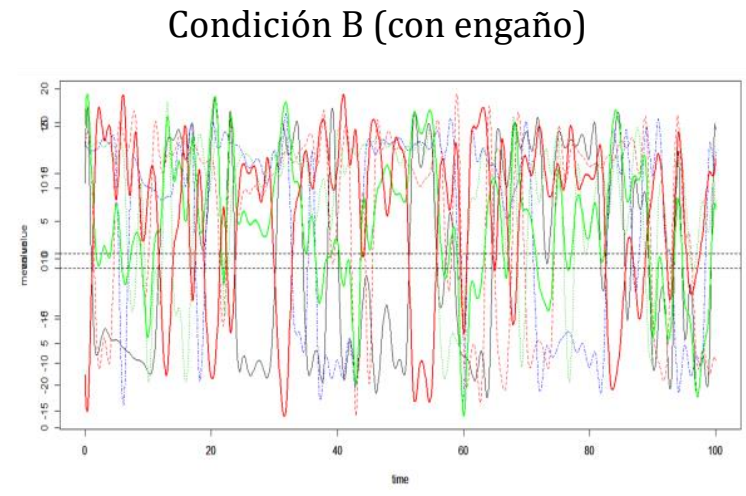

Respecto a la condición B de la Figura 3 , la media funcional se encuentra entre valores de $-10 \mu \mathrm{V}$ y $15 \mu \mathrm{V}$ (donde los valores negativos de la media corresponden a fluctuaciones o disparos eléctricos extremos de la onda. Esto implica que los datos de la media funcional son más constantes entre 0 y $15 \mu \mathrm{V}$ ). En contraste, la desviación estándar funcional presenta valores entre $0,5 \mu \mathrm{V}$ y $2 \mu \mathrm{V}$ con una variación significativa de $4 \mu \mathrm{V}$, esto indica que los datos presentan mayores índices de dispersión que en la condición sin engaño.

\section{Figura 4}

Actividad eléctrica de la corteza frontal, electrodo F3

Condición A (sin engaño)

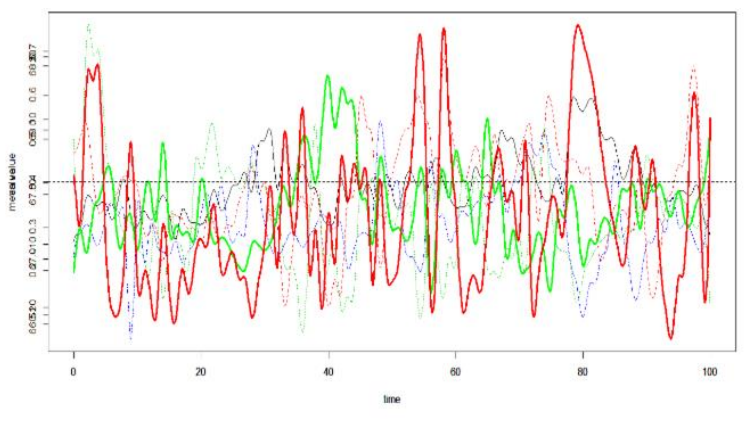

Condición B (con engaño)

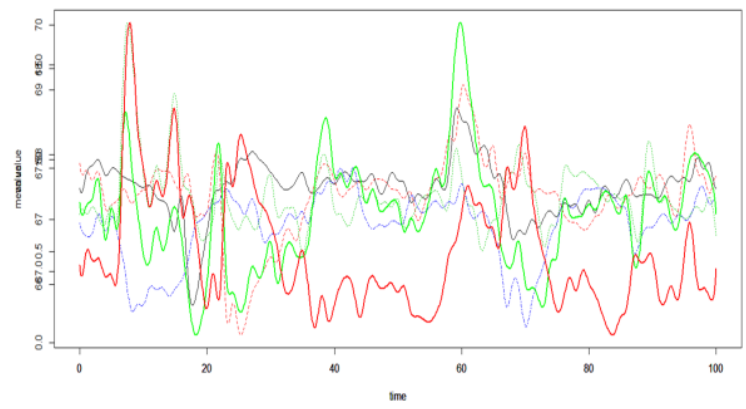


La actividad eléctrica del electrodo F3, representado en la Figura 4, muestra que durante la condición $\mathrm{A}$, el rango de amplitud entre $65 \mu \mathrm{V}$ y $69 \mu \mathrm{V}$ con un pico de $62 \mu \mathrm{V}$, lo cual indica que la onda en general presenta una polaridad positiva con un comportamiento estable entre $66 \mu \mathrm{V}$ y $68 \mu \mathrm{V}$ en las dos condiciones. Sin embargo, los valores de la media y desviación estándar en la condición sin engaño muestran bajos índices de variabilidad respecto a la condición B. No se evidencian cambios significativos en los niveles de ruido de las ondas, por el contrario, se observa un patrón estable en la actividad eléctrica cerebral.

Respecto a la condición B el nivel de voltaje presenta una menor polaridad positiva en comparación a la condición A, presentando índices de voltaje entre $67 \mu \mathrm{Vy}$ $68 \mu \mathrm{V}$, con un descenso en la onda entre $65 \mu \mathrm{V}$ y $67 \mu \mathrm{V}$ cerca al rango 500 del eje $\mathrm{x}$, descenso que se recuperara de forma gradual, lo indica un movimiento progresivo en la actividad cerebral.

\section{Figura 5}

Actividad eléctrica de la corteza frontal, electrodo F4 Condición A (sin engaño)

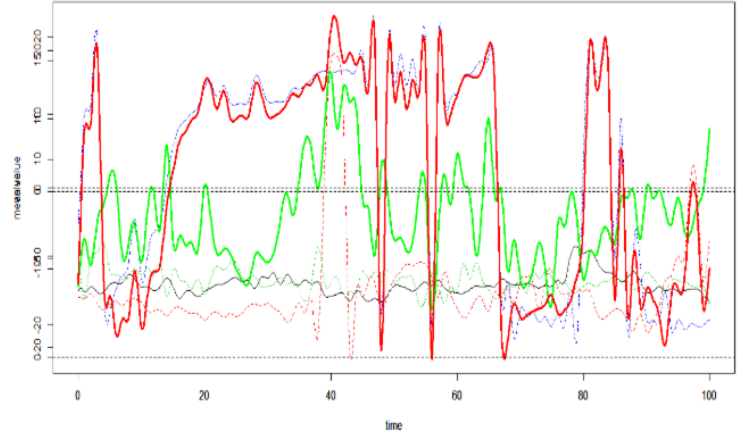

La Figura 5, correspondiente al electrodo F4, (gráfica A), muestran amplitudes en la onda con valores negativos y positivos que van desde los -20 $\mu \mathrm{V}$ a $20 \mu \mathrm{V}$, no obstante, se observa que el promedio de las curvas se mantiene entre los $-10 \mu \mathrm{V}$, lo cual indica que existe gran dispersión de los datos a lo largo de la medición. A pesar de que se observan características de rugosidad en el segmento inicial de las curvas, los cuales se acompañan de variaciones descendentes de la actividad eléctrica al inicio y en el intermedio de la medición.
Condición B (con engaño)

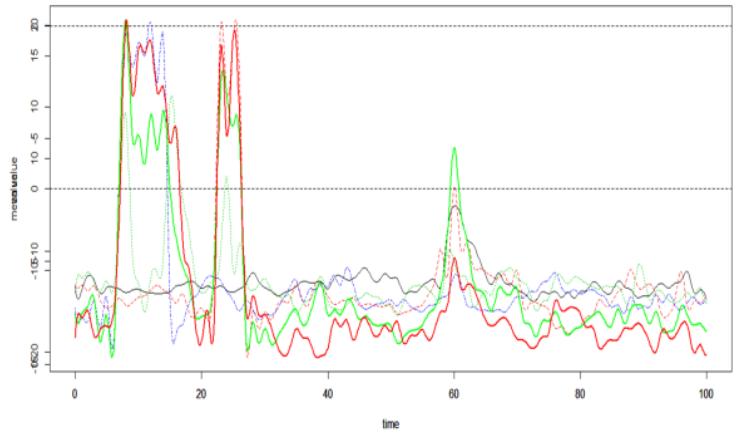

Es importante resaltar que en la condición B, se observan alteraciones ascendentes de la actividad eléctrica al iniciar la condición de engaño, las cuales tienden a estabilizarse en los segmentos posteriores durante la medición. Adicionalmente, la distancia entre la curva media y la desviación estándar mantienen una relación proporcional, lo cual representa un grado bajo de dispersión entre las curvas. La medición no registró presencia de datos atípicos tanto en la condición A como en la condición B. 
Figura 6

Actividad eléctrica de la corteza frontal, electrodo F8 Condición A (sin engaño) Condición B (con engaño)
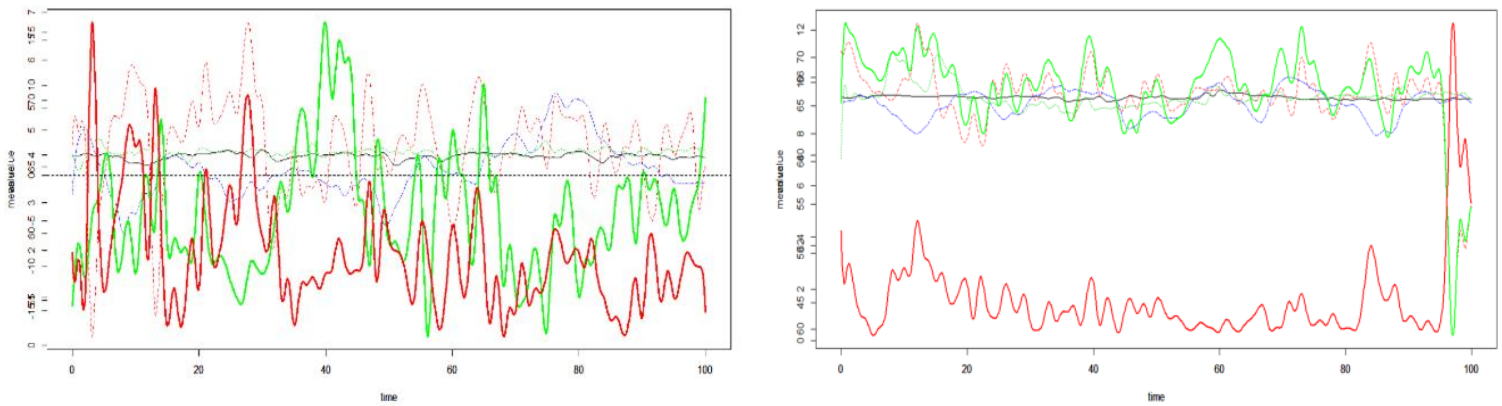

En la Figura 6 se observa que la señal eléctrica correspondiente a la condición $\mathrm{A}$, presenta un rango de amplitud de $55 \mu \mathrm{V}$ a $75 \mu \mathrm{V}$, mostrando un patrón de la actividad eléctrica con polaridad positiva. Por otra parte, se observa que la magnitud de la desviación estándar es mucho más baja que la media, lo cual indica niveles bajos de dispersión en la actividad eléctrica de los sujetos durante la condición sin engaño.

Respecto en la condición B, se aprecia que el rango de la amplitud oscila entre 45 $\mu \mathrm{V}$ a $70 \mu \mathrm{V}$, incrementando $5 \mu \mathrm{V}$ con

respecto a la condición A. Por otra parte, pese a que la media funcional tiende a estabilizarse, se observa un distanciamiento respecto a la curva de la desviación típica, indicando niveles superiores en la amplitud de la señal eléctrica para la mayoría de los sujetos, por lo tanto, la desviación estándar es considerablemente baja en comparación a la media funcional. Cambios en el comportamiento de la desviación estándar al final de la medición indicaron la presencia de datos atípicos en la condición B.

\section{Figura 7}

Actividad eléctrica de la corteza frontal, electrodo FC5

Condición A (sin engaño)

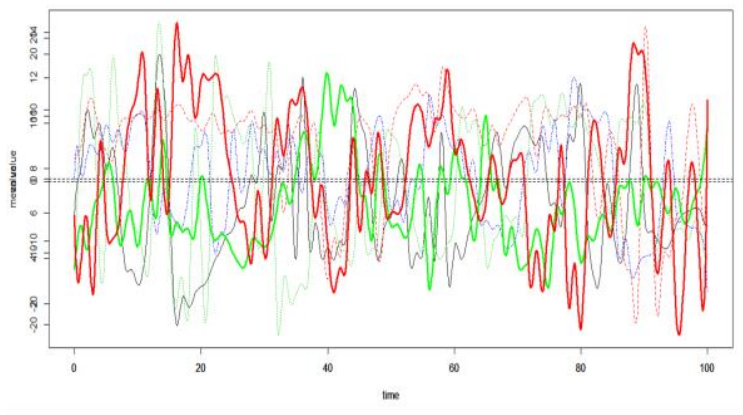

Condición B (con engaño)

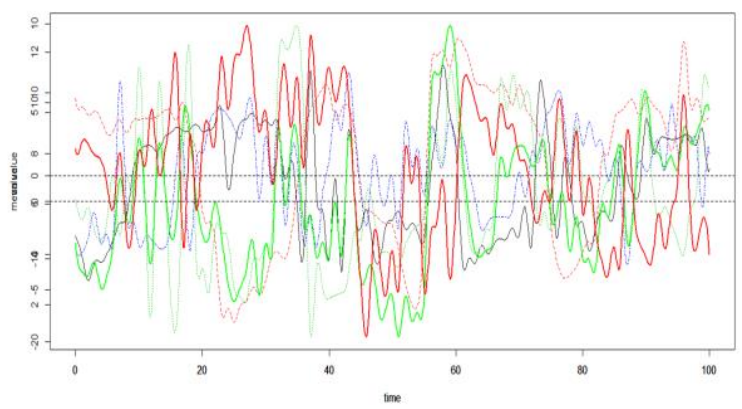


La actividad eléctrica registrada por el electrodo FC5, Figura 7, muestra un comportamiento inestable caracterizado por una continua variabilidad a lo largo de la longitud de la onda, la cual presenta polaridad positiva y negativa con un rango de amplitud que va desde $-20 \mu \mathrm{V}$ a $20 \mu \mathrm{V}$. A pesar de que no se observaron datos atípicos, los cambios entre la desviación estándar y la media funcional indican niveles altos de rugosidad y dispersión de los datos en ambas condiciones. Por otra parte, en la condición B se observan niveles de amplitud y polaridad similares a la condición inicial, puesto que el rango observado se mantiene entre los $-20 \mu \mathrm{V}$ a 20 $\mu \mathrm{V}$. Respecto al grado de rugosidad se identifican cambios recurrentes entre la media y la desviación estándar, mostrando niveles superiores en la dispersión de los datos en comparación a la condición A.

\section{Figura 8}

Actividad eléctrica de la corteza frontal, electrodo FC6

Condición A (sin engaño)

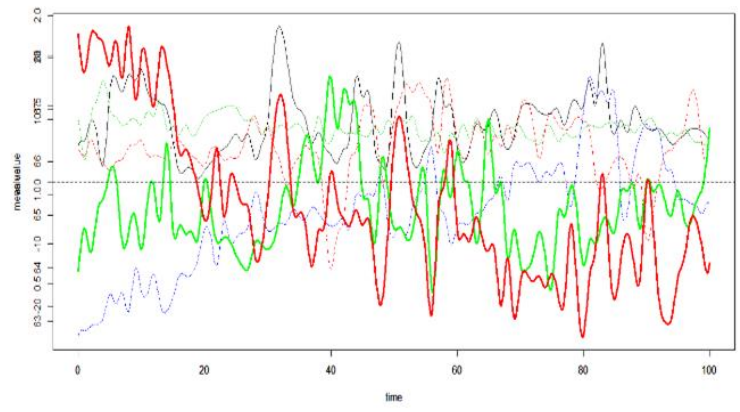

La medición observada Figura 8, mostró un rango de amplitud que va desde $63 \mu \mathrm{V}$ a $68 \mu \mathrm{V}$, lo cual indica que la actividad eléctrica registrada por este electrodo mantuvo una polaridad positiva constante durante la medición. En términos de la estabilidad de la onda, se observa que las curvas se mantienen dentro del intervalo de confianza, presentando niveles moderados de aglomeración o rugosidad de las curvas y nulidad de datos atípicos. A pesar de que al inicio de la condición A se observó mayor variabilidad en la actividad eléctrica cerebral, a nivel general la medición tuvo una mayor concentración en relación con la curva media, indicando bajos niveles en la

\section{Condición B (con engaño)}

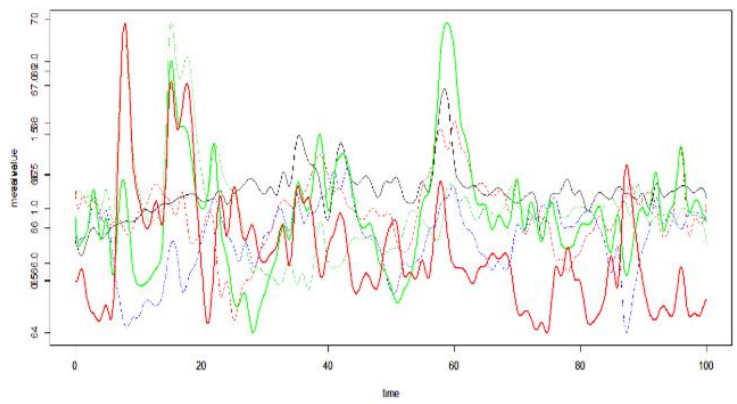

dispersión de los datos, particularmente entre $65 \mu \mathrm{V}$ a $67 \mu \mathrm{V}$.

Por otro lado, los resultados obtenidos durante la condición de engaño muestran un menor grado en la variabilidad de las curvas, es decir que presentan menos alteraciones en términos de la curva media, sin embargo, se observa que la señal eléctrica aumentó moderadamente respecto a la condición $\mathrm{A}$, estableciendo un rango entre los $70 \mu \mathrm{V}$ a $64 \mu \mathrm{V}$. De igual forma se observa que pese a que se presentan picos elevados, esto no modificó la rugosidad ni la dispersión de los datos. 


\section{Discusión}

Los resultados indicaron que en la condición B (con engaño), las ondas cerebrales tienden a ser más estables, lo cual indica que en situaciones de engaño hay una menor variabilidad de la señal eléctrica cerebral. Por otra parte, en la condición A (sin engaño), las curvas tendieron a ser menos estables, rugosas y con mayor variabilidad a pesar de encontrarse bajo menor amplitud. De acuerdo con Tirapu-Ustárroz, Pérez-Sayes, Erekatxo-Bilbao, y Pelegrín-Valero, (2007), la activación de áreas asociadas con el acceso a la memoria semántica y planificación, incluyendo el giro frontal medial izquierdo y cingulado, presentan una mayor activación eléctrica en la elaboración de mentiras, razón por la cual se explica la presencia de conexiones con múltiples áreas del lóbulo frontal y los procesos ejecutivos subyacentes a la capacidad de regulación, supervisión y elección de una conducta con fines de engaño (Battista et al., 2021).

En términos generales se observó mayor amplitud en las ondas bajo la condición B (con engaño), oscilando desde $20 \mu \mathrm{V}$ hasta $78 \mu \mathrm{V}$, en tanto que bajo la condición A (sin engaño) se presentó una oscilación de la onda desde $-20 \mu \mathrm{V}$ hasta 69 $\mu \mathrm{V}$, es claro que bajo la condición $\mathrm{B}$ hubo mayor activación de las áreas frontotemporales, reportando mayor actividad eléctrica cerebral de manera global. Por su parte, la actividad eléctrica correspondiente a la circunvolución frontal inferior o también conocida como la región orbitaria, presenta características relacionadas con procesos vegetativos primitivos, tales como: la activación de la respuesta de alarma, el control de la respiración, presión arterial, lo cual plantea una relación estrecha con los factores emocionales implícitos en la conducta de engaño, razón por la cual es posible inferir que los procesos de inhibición de la información verdadera, pueden estar relacionados con la percepción de beneficio ante una potencial situación de amenaza (Blandón-Gitlin, López, Masip, y Fenn, 2017).

Según Jodar-Vicente (2004) la corteza prefrontal compuesta por los electrodos AF3 y AF4, se encarga de organizar secuencias de comportamientos y toma de decisiones dirigidas a la consecución de objetivos específicos, los cuales incluyen movimientos oculares, razonamiento y valoraciones emocionales, lo cual explica el amplio incremento de la actividad eléctrica durante la fase de medición y registro electroencefalográfico en la condición de engaño. Por otra parte, los electrodos FC5, FC6 y F8, encargados de registrar las señales provenientes de las áreas de Brodman 44 y 45, correspondientes al área de Broca, mostraron niveles de activación superior, teniendo en cuenta que se reconoce una función articulatoria del lenguaje, la cual presenta una mayor simetría en el hemisferio izquierdo. Cabe resaltar que las variaciones entre la actividad positiva y negativa que mostró el electrodo FC5 podría estar relacionada con la función inhibitoria al momento de elaborar una mentira (Karim, Schneider, Lotze, Veit, Sauseng, Braun y Birbaumer, 2010). 
Respecto a los electrodos F3 y F4 Monterroso y Avilez (2008) afirman que se encuentran ubicados en el área motora suplementaria AMS, que junto con el área motora primaria se encargan de la actividad motora presente en la expresión no verbal. Según estos autores, cuando se activa mentalmente una secuencia de movimientos sin su respectiva elaboración, se observa una mayor actividad del AMS, por tanto, se considera que este sector se encarga especialmente de la planeación estratégica de los movimientos, lo cual resulta consistente con la manifestación no verbal del engaño, puesto que, según Ludwig, Van Laer, De Ruyter y Friedman, (2016), los factores asociados a la comunicación, la empatía y la intencionalidad son fundamentales al momento de mentir o engañar.

\section{Conclusiones}

El presente estudio tuvo como objetivo identificar características de la actividad eléctrica cerebral generada en condiciones de sinceridad y engaño en estudiantes universitarios, a través de la aplicación de un modelo funcional para el análisis de datos electrofisiológicos. A nivel general, es posible considerar que el análisis funcional mostró ser apropiado estadísticamente para procesar información relacionada con la actividad eléctrica cerebral usando el equipo EmotivEpoc, lo cual sugiere que, a nivel descriptivo, el modelo puede ser usado para identificar diferencias entre los patrones de la actividad neuronal asociada con la declaración de información real y ficticia en jóvenes universitarios.
Adicionalmente, esta investigación proporcionó una aproximación cuantitativa novedosa aplicada al estudio del comportamiento eléctrico del cerebro y las funciones cognitivas subyacentes al procesamiento de información autobiográfica en jóvenes adultos. La exploración de los datos recolectados conlleva a preguntas más complejas, asociadas a la efectividad de nuevos modelos para hacer inferencias que den respuesta a eventos observables de la actividad cerebral cuando se realizan tareas cognitivas específicas con el fin de identificar correlatos neuronales.

Cabe resaltar que futuros estudios son requeridos para determinar diferencias significativas entre variables sociodemográficas y culturales. Adicionalmente, se sugiere incluir un número representativo de la población seleccionada, con el propósito de confirmar que la aplicación del análisis descriptivo funcional puede ser generalizado y por consiguiente, poder implementar algoritmos basados en los modelos inferenciales funcionales que permitan predecir cambios bioeléctricos en la actividad neuronal de la corteza frontal y áreas de procesamiento cognitivo subyacente a la elaboración de mentiras. 


\section{Referencias}

Battista, F., Otgaar, H., Mangiulli, I., \& Curci, A. (2021). The role of executive functions in the effects of lying on memory. Acta Psychologica, 215, 103295.

https://www.sciencedirect.com/sc ience/article/pii/S0001691821000 457

Berberyan, H. S., van Maanen, L., van Rijn, H., \& Borst, J. (2021). Eeg-based identification of evidence accumulation stages in decisionmaking. Journal of Cognitive Neuroscience, 33(3), 510-527. https://direct.mit.edu/jocn/articleabstract/33/3/510/95553/EEGbased-Identification-of-EvidenceAccumulation

Bessadok, A., Mahjoub, M. A., \& Rekik, I. (2021). Graph Neural Networks in Network Neuroscience. arXiv preprint arXiv:2106.03535. https://arxiv.org/abs/2106.03535

Blandón-Gitlin, I., López, R. M., Masip, J., \& Fenn, E. (2017). Cognición, emoción y mentira: implicaciones para detectar el engaño. Anuario de Psicología Jurídica, 27(1), 95-106.

Castro, C., López, y Morales, M. (2013). Estudio Cognitivo de la mentira humana. Ciencia UANL, 16(64), 91102.

http://eprints.uanl.mx/7088/1/Es tudio-cognitivo-de-la-mentira.pdf

Cortes, P. M., García-Hernández, J. P., IribeBurgos, F. A., Hernández-González,
M., Sotelo-Tapia, C., \& Guevara, M. A. (2021). Temporal division of the decision-making process: An EEG study. Brain Research, 1769, 147592.

https://www.sciencedirect.com/sc ience/article/abs/pii/S000689932 1004492

Gutiérrez, M., Giner, P., Quintero, K., Moret, C. y Moliner, J. (2014). Estudio piloto sobre las huellas cerebrales durante el engaño simulado. Revista iberoamericana interdisciplinar de métodos, modelización y simulación, 7 , 19-26. https://dialnet.unirioja.es/servlet/ articulo?codigo $=5137422$

Ierache, J. S., Pereira, G., Iribarren, J., \& Nervo, F. (2014). Estado emocional centrado en estímulos, aplicando Interfase cerebro-máquina. In $X X$ Congreso Argentino de Ciencias de la Computación (Buenos Aires, 2014). http://sedici.unlp.edu.ar/handle/1 0915/42260

Jodar-Vicente, M. (2004). Funciones cognitivas del lóbulo frontal. Revista de neurología, 39(2), 178 - 182. https://pesquisa.bvsalud.org/porta l/resource/pt/ibc-34481

Karim, A. A., Schneider, M., Lotze, M., Veit, R., Sauseng, P., Braun, C., \& Birbaumer, N. (2010). The truth about lying: inhibition of the anterior prefrontal cortex improves deceptive behavior. Cerebral cortex (New York, N.Y.: 1991), 20(1), 205-213. 
https://doi.org/10.1093/cercor/b hp090

Kouhbanani, S. S., \& Arabi, S. M. (2021). Home executive function environment and executive functions in children: The mediating role of brain electrical activity. Current Psychology, 1-9. https://link.springer.com/article/1 0.1007/s12144-021-02044-4

Ludwig, S., Van Laer, T., De Ruyter, K., \& Friedman, M. (2016). Untangling a web of lies: Exploring automated detection of deception in computermediated communication. Journal of Management Information Systems, 33(2), 511-541. https://www.tandfonline.com/doi /full/10.1080/07421222.2016.120 5927

Maestú, F., Quesney-Molina, F., OrtizAlonso, T., Campo, P., FernándezLucas, A., \& Amo, C. (2003). Cognición y redes neuronales: una nueva perspectiva desde la neuroimagen funcional. Rev Neurol, 37(10), 962-6. https://doi.org/10.33588/rn.3710. 2003069

Martínez Luquero, M. D. C. (2020). Diseño y evaluación de la efectividad de una nueva modalidad de entrevista policial para detectar mentiras [Tesis doctoral, Universidad de Salamanca]. http://hdl.handle.net/10366/1455 32
Monaro, M., Maldera, S., Scarpazza, C., Sartori, G., \& Navarin, N. (2022). Detecting deception through facial expressions in a dataset of videotaped interviews: A comparison between human judges and machine learning models. Computers in Human Behavior, 127, 107063.

Monterroso, E. M., Avilez, A. B., \& Vanegas, M. A. A. (2008). Área motora suplementaria. Archivos de Neurociencias, 13(2), 118-124.

Ortega González, M. (2010). Comportamiento mentiroso: Un análisis conceptual desde una perspectiva interconductual. Revista electrónica de psicología Iztacala, 13(1) https://www.iztacala.unam.mx/car reras/psicologia/psiclin/vol13num 1/Art5Vol13No1.pdf

Piñerez, W. J. R., Ramírez, A. C., \& Escobar, 0. G. (2017). Análisis de datos funcionales aplicado en electroencefalogramas: agrupamiento por k-medias funcional. Comunicaciones en Estadística, 10(1), 129-144. https://dialnet.unirioja.es/servlet/ articulo?codigo $=6765740$

Ramsay, J. y Silverman, B. (2002). Applied functional data analysis: Methods and case studies. Springer.

Rodríguez-Valdez, R., Manrique-Suárez, V., Álvarez-Amador, A., Galán-García, L., Fernández-García, Y., LópezCánovas, L., Riverón, A. Bobes-León, 
M. (2008). Análisis Espectral del Electroencefalograma en Sujetos con Deterioro Cognitivo Leve. Revista de Neurología, 46(5), 267272.

https://www.researchgate.net/pro file/VianaSuarez/publication/5499983_Spec tral_analysis_of_the_electroencepha logram_in_subjects_with_mild_cogn itive_impairment/links/55d35063 08ae7fb244f58646/Spectralanalysis-of-theelectroencephalogram-in-subjectswith-mild-cognitiveimpairment.pdf

Sahakian, B. J., \& Gottwald, J. (2016). Sex, Lies, and Brain Scans: How fMRI reveals what really goes on in our minds. Oxford University Press.

Shaughnessy, J., Zechmeister, E. y Shaughnessy, J. (2007). Métodos de investigación en psicología. McGraw-Hill

Tirapu-Ustárroz, J., García-Molina, A., LunaLario, P., Roig-Rovira, T., \& PelegrínValero, C. (2008). Modelos de funciones y control ejecutivo (I). Revista de neurología, 46(11), 684 692.

https://pavlov.psyciencia.com/201 2/10/Modelos-de-funciones-ycontrol-ejecutivo.pdf. 